\title{
Geometric Graphs with Few Disjoint Edges*
}

\author{
G. Tóth ${ }^{1,2}$ and P. Valtr ${ }^{2,3}$ \\ ${ }^{1}$ Courant Institute, New York University, \\ 251 Mercer Street, New York, NY 10012, USA \\ toth@cims.nyu.edu \\ 2 DIMACS Center, Rutgers University, \\ Piscataway, NJ 08855, USA \\ ${ }^{3}$ Department of Applied Mathematics, Charles University, \\ Malostranské nám. 25, 11800 Praha 1, Czech Republic \\ valtr@kam.mff.cuni.cz
}

\begin{abstract}
A geometric graph is a graph drawn in the plane so that the vertices are represented by points in general position, the edges are represented by straight line segments connecting the corresponding points.

Improving a result of Pach and Törőcsik, we show that a geometric graph on $n$ vertices with no $k+1$ pairwise disjoint edges has at most $k^{3}(n+1)$ edges. On the other hand, we construct geometric graphs with $n$ vertices and approximately $\frac{3}{2}(k-1) n$ edges, containing no $k+1$ pairwise disjoint edges.

We also improve both the lower and upper bounds of Goddard, Katchalski, and Kleitman on the maximum number of edges in a geometric graph with no four pairwise disjoint edges.
\end{abstract}

\section{Introduction}

A geometric graph $G$ is a graph drawn in the plane by (possibly crossing) straight line segments, i.e., it is defined as a pair $G=(V, E)$, where $V$ is a set of points in general position in the plane and $E$ is a set of closed segments whose endpoints belong to $V$.

The following problem was raised by Avital and Hanani [AH], Kupitz [K], Erdôs, and Perles. Determine the smallest number $e_{k}(n)$ such that any geometric graph with $n$ vertices and $m>e_{k}(n)$ edges contains $k+1$ pairwise disjoint edges. By a result

\footnotetext{
* G. Tóth was supported by OTKA-T-020914, OTKA-F-22234 and NSF Grant CCR-94-24398. P. Valtr was supported by the Czech Republic Grant GAČR 201/1996/0194 and Charles University Grants GAUK $193,194 / 1996$.
} 
of Hopf and Pannwitz [HP] and Erdős, $e_{1}(n)=n$. Alon and Erdős [AE] showed that $e_{2}(n) \leq 6 n-5$ which was improved by Goddard et al. [GKK] to $e_{2}(n) \leq 3 n$. The best known lower bound, $e_{2}(n) \geq 2.5 n-4$, is due to Perles (see [PA] and [MP]). It has also been known that $3.5 n-6 \leq e_{3}(n) \leq 10 n$ and that $e_{k}(n) \leq c_{k} n(\log n)^{k-3}(k \geq 4)$, see [GKK]. For any fixed $k$, Pach and Töröcsik [PT] were first to prove that $e_{k}(n)$ is linear in $n$; their bound was $e_{k}(n) \leq k^{4} n$. It follows from a result of Kupitz [K] that $e_{k}(n) \geq k n$. In this paper we further improve both the upper and lower bounds for general $k$.

Theorem 1. For $k \leq n / 2$,

$$
\frac{3}{2}(k-1) n-2 k^{2} \leq e_{k}(n) \leq k^{3}(n+1) .
$$

We also improve the above mentioned bounds on $e_{3}(n)$.

Theorem 2. For any $n \geq 6$,

$$
4 n-9 \leq e_{3}(n) \leq 8.5 n \text {. }
$$

Theorems 1 and 2 are proved in Sections 2 and 3, respectively. Throughout the paper, we do not make any notational distinction between an edge and the segment representing it.

Very recently, using similar methods, Tóth has further improved the upper bound in Theorem 1 to $e_{k} \leq 2^{9} k^{2} n$ [T]. Two related problems were studied in [LMPT] and [V]. In [V] it was shown that the number of edges in a geometric graph with no $k$ pairwise crossing edges is at most $c_{k} n \log n$. In [LMPT] it was proved that among any $k^{5}$ convex sets in the plane, one can find $k$ of them which are either pairwise disjoint or pairwise intersecting (in particular, this also holds for line segments).

\section{The General Case}

\subsection{The Upper Bound}

Our proof, as the proof of Pach and Törôcsik [PT], is based on Dilworth's theorem [D].

Dilworth's Theorem. Let $P$ be a partially ordered set containing no chain (totally ordered subset) of size $k+1$. Then $P$ can be covered by $k$ antichains (subsets of pairwise incomparable elements).

Let $G=(V, E)$ be a geometric graph on $n$ vertices, containing no $k+1$ pairwise disjoint edges. For a vertex $v$, let $x(v)$ and $y(v)$ denote its $x$ - and $y$-coordinate, respectively. We can assume without loss of generality that no two vertices have the same $x$-coordinate.

An edge $e$ is said to lie below an edge $e^{\prime}$, if no vertical line crossing both $e$ and $e^{\prime}$ crosses $e$ strictly above $e^{\prime}$. Finally, let $\pi(e)$ denote the orthogonal projection of $e$ to the $x$-axis. 


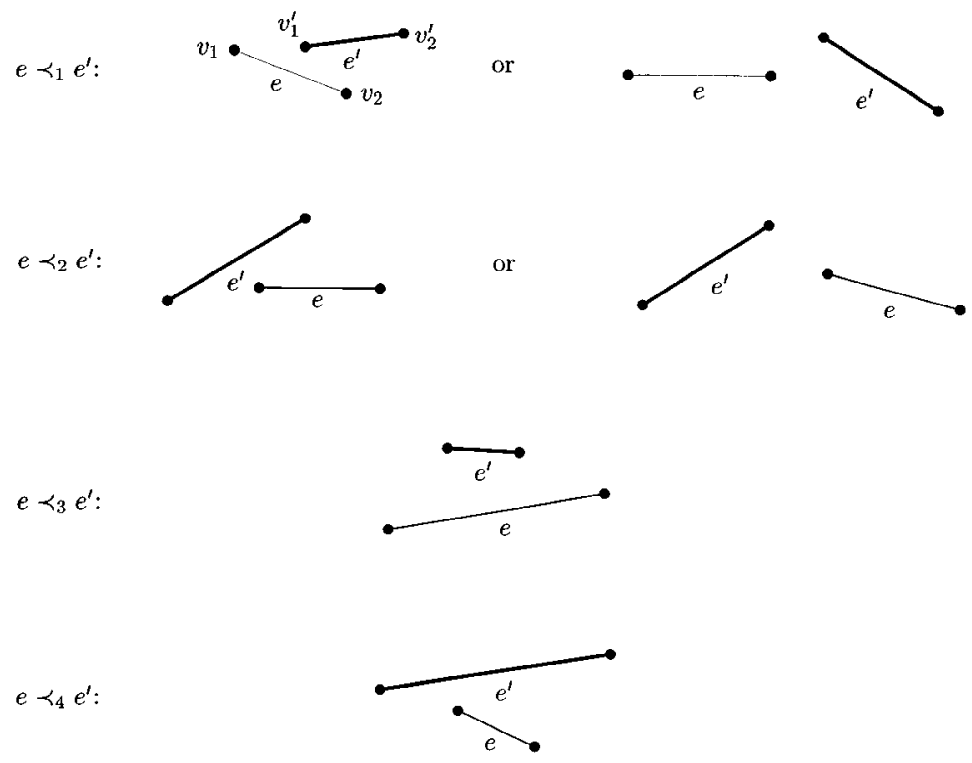

Fig. 1. The relations $\prec_{i}$.

Define four binary relations $\prec_{i}(i=1, \ldots, 4)$ on the edge set $E$ as follows (see also [PT] and [PA]). Let $e=v_{1} v_{2}$ and $e^{\prime}=v_{1}^{\prime} v_{2}^{\prime}$ be two disjoint edges of $G$, where $x\left(v_{1}\right)<x\left(v_{2}\right)$ and $x\left(v_{1}^{\prime}\right)<x\left(v_{2}^{\prime}\right)$. Then (see Fig. 1)

$$
\begin{array}{llll}
e \prec_{1} e^{\prime}, & \text { if } \quad x\left(v_{1}\right)<x\left(v_{1}^{\prime}\right), & x\left(v_{2}\right)<x\left(v_{2}^{\prime}\right), & \text { and } e \text { lies below } e^{\prime}, \\
e \prec_{2} e^{\prime}, & \text { if } \quad x\left(v_{1}\right)>x\left(v_{1}^{\prime}\right), & x\left(v_{2}\right)>x\left(v_{2}^{\prime}\right), & \text { and } e \text { lies below } e^{\prime}, \\
e \prec_{3} e^{\prime}, & \text { if } x\left(v_{1}\right)<x\left(v_{1}^{\prime}\right), & x\left(v_{2}\right)>x\left(v_{2}^{\prime}\right), & \text { and } e \text { lies below } e^{\prime}, \\
e \prec_{4} e^{\prime}, & \text { if } \quad x\left(v_{1}\right)>x\left(v_{1}^{\prime}\right), & x\left(v_{2}\right)<x\left(v_{2}^{\prime}\right), & \text { and } e \text { lies below } e^{\prime} .
\end{array}
$$

Obviously, each of the relations $\prec_{i}$ is a partial ordering, and any pair of disjoint edges in $G$ is comparable by at least one of them.

Since $G$ does not contain $k+1$ disjoint edges, $\left(E, \prec_{1}\right)$ does not contain a chain of length $k+1$. Therefore, by Dilworth's theorem, $E$ can be covered by $k$ antichains with respect to $\prec_{1}$. Let $E_{1}$ be the largest of these antichains, thus $\left|E_{1}\right| \geq|E| / k$. Applying Dilworth's theorem on $\left(E_{1}, \prec_{2}\right)$, we similarly get an antichain (with respect to $\prec_{2}$ ) $E_{2} \subseteq E_{1}$ of size $\left|E_{2}\right| \geq\left|E_{1}\right| / k \geq|E| / k^{2}$. In the rest of the proof we estimate the size of $E_{2}$ from above.

Since $E_{2}$ is an antichain with respect to $\prec_{1}$ and $\prec_{2}, \pi(e) \cap \pi\left(e^{\prime}\right) \neq \emptyset$ for any $e, e^{\prime} \in E_{2}$. Therefore, $\bigcap_{e \in E_{2}} \pi(e) \neq \emptyset$, so there is a vertical line $\ell$ which intersects all edges in $E_{2}$.

Let $\overrightarrow{G_{2}}=\left(V, \overrightarrow{E_{2}}\right)$ be a directed geometric graph obtained from $\left(V, E_{2}\right)$ by replacing each edge $e=v_{1} v_{2}$ in $E_{2}$ by the two oriented edges $\overrightarrow{v_{1} v_{2}}$ and $\overrightarrow{v_{2} v_{1}}$. For two edges $e_{1}=\overrightarrow{v_{0} v_{1}}, e_{2}=\overrightarrow{v_{1} v_{2}}$ forming a path in $\vec{G}_{2}$, we say that $e_{2}$ is a zag of $e_{1}$, if the following 


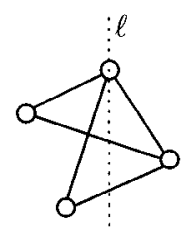

$G_{2}$
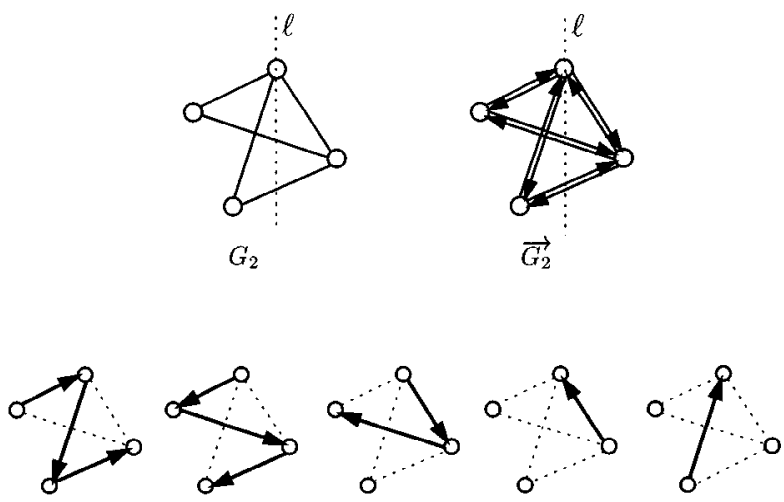

all 5 maximal zigzag paths in $\overrightarrow{G_{2}}$

Fig. 2. Maximal zigzag paths.

two conditions hold:

(i) $\pi\left(e_{1}\right) \cap \pi\left(e_{2}\right)$ has positive length,

(ii) for any $z \in\left(\pi\left(e_{1}\right) \cap \pi\left(e_{2}\right)\right) \backslash\left\{\pi\left(v_{1}\right)\right\}$, the vertical line through $z$ intersects $e_{2}$ below $e_{1}$, and it intersects no other edge going from $v_{1}$ between $e_{1}$ and $e_{2}$.

Observe that each edge in $\overrightarrow{G_{2}}$ has at most one zag. We call an oriented path $e_{1} e_{2} \cdots e_{r}$ in $\vec{G}_{2}$ a zigzag path, if $e_{i+1}$ is the zag of $e_{i}$, for each $i=1, \ldots, r-1$ (see Fig. 2).

Lemma 3. Every zigzag path in $\vec{G}_{2}$ has at most $2 k$ edges.

Lemma 4. $\overrightarrow{G_{2}}$ has at most $n+1$ maximal zigzag paths.

Lemmas 3 and 4 immediately give Theorem 1: every edge in $\vec{G}_{2}$ is contained in a maximal zigzag path; therefore, by Lemmas 3 and 4,

$$
\left|\overrightarrow{E_{2}}\right| \leq 2 k(n+1)
$$

and, consequently,

$$
|E| \leq k^{2}\left|E_{2}\right|=k^{2}\left|\vec{E}_{2}\right| / 2 \leq k^{3}(n+1) .
$$

It remains to prove Lemmas 3 and 4.

Proof of Lemma 3. Let $P=e_{1} e_{2} \cdots e_{r}$ be a zigzag path in $\vec{G}_{2}$, and let $e_{i}={\overrightarrow{v_{i-1}}}_{i}$ for $i=1, \ldots, r$. We need the following claim.

Claim. One of the two sequences

$$
S_{0}=x\left(v_{0}\right), x\left(v_{2}\right), \ldots, \quad S_{1}=x\left(v_{1}\right), x\left(v_{3}\right), \ldots
$$

is decreasing, and the other one is increasing. 

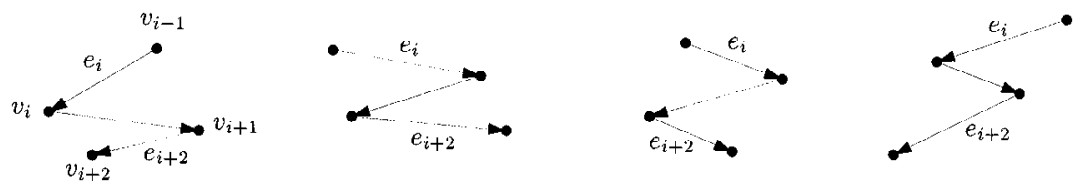

Fig. 3. $e_{i+2} \prec_{1} e_{i}$ or $e_{i+2} \prec_{2} e_{i}$.

Proof of the Claim. Suppose the claim is false. Then there is an index $i$ such that either $x\left(v_{i-1}\right)<x\left(v_{i+1}\right)$ and $x\left(v_{i}\right)<x\left(v_{i+2}\right)$, or $x\left(v_{i-1}\right)>x\left(v_{i+1}\right)$ and $x\left(v_{i}\right)>x\left(v_{i+2}\right)$. Consequently, $e_{i+2} \prec_{2} e_{i}$ or $e_{i+2} \prec_{1} e_{i}$ (see Fig. 3 for all four possible cases), which is a contradiction with the definition of $E_{2}$. The claim is proved.

The length of $P$ is at most $2 k$ since otherwise $e_{1}, e_{3}, \ldots, e_{2 k+1}$ would be $k+1$ disjoint edges according to the Claim (see Fig. 4).

Proof of Lemma 4. Let $v \in V$ be a vertex lying to the right of $\ell$, and let $P_{1}, P_{2}$ be two different zigzag paths in $\vec{G}_{2}$ ending in $v$. If the slope of the last edge in $P_{1}$ is, say, smaller than the slope of the last edge in $P_{2}$, then the last edge of $P_{1}$ has a zag and, consequently, $P_{1}$ can be extended to a longer zigzag path. It follows that at most one maximal zigzag path ends in $v$, and this is similarly true for any vertex not lying on $\ell$. Similarly, at most two zigzag paths end in any vertex $v$ on $\ell$ (one coming to $v$ from the left, the other one from the right). The lemma now follows from the assumption that no pair of vertices lies on a vertical line.

\subsection{The Lower Bound}

For simplicity, suppose that $k$ is even and $n$ is odd. Set $z=(n-k+1) / 2$. Let $P$ be a set of $z$ points $p_{1}, \ldots, p_{z}$ placed equidistantly in this order from left to right on a horizontal line $p$. Let $Q=\left\{q_{1}, \ldots, q_{z}\right\}$ be a translation of $P$ such that $q_{i}$ always corresponds to $p_{i}$ and $p_{1} p_{z} q_{z} q_{1}$ is a square. Let $q$ be the line containing $Q$, and let $r$ be the line parallel
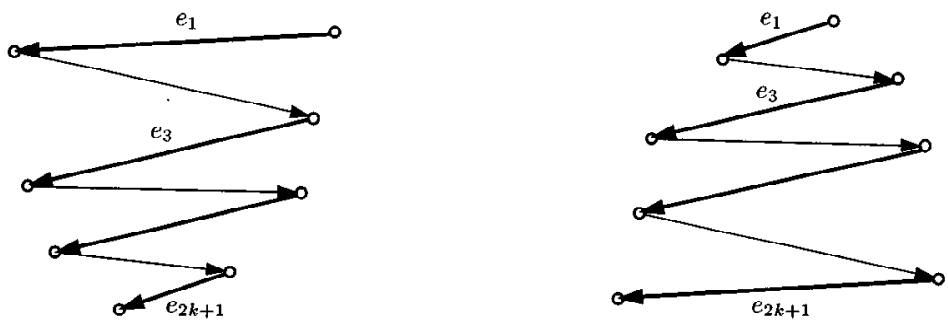

Fig. 4. $e_{1}, e_{3}, \ldots, e_{2 k+1}$ are disjoint. 


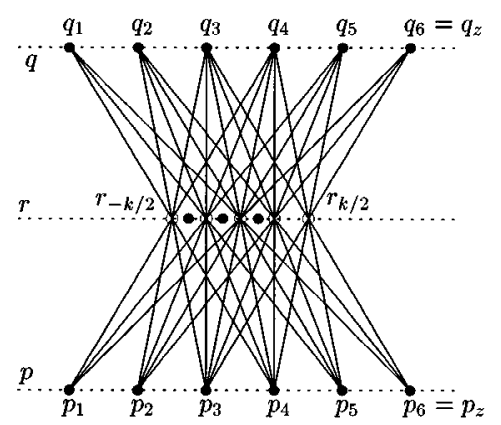

$E_{1}^{\top}$

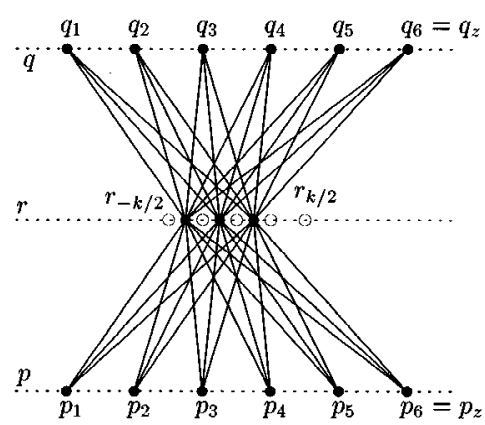

$E_{2}$

Fig. 5. The edges of $E_{1}$ and $E_{2}$ (for $k=4, n=15, z=6$ ).

to $p$ and $q$, halfway between them. Let $E_{1}$ be the set of all segments (edges) $p_{i} q_{j}$ with $-k / 2 \leq i+j-(z+1) \leq k / 2$ (see Fig. 5).

The segments of $E_{1}$ intersect the line $r$ in $k+1$ points $r_{-k / 2}, r_{-k / 2+1}, \ldots, r_{k / 2}$ such that $r_{t}$ always lies on the segments $p_{i} q_{j}$ with $i+j-(z+1)=t$. Let $R$ be the set of the centers of the segments $r_{t} r_{t+1}(t=-k / 2,-k / 2+1, \ldots, k / 2-2)$, and let $E_{2}$ be the set of all edges (segments) joining vertices of $R$ with vertices of $P \cup Q$ (see Fig. 5).

We now show that the geometric graph $G=\left(P \cup Q \cup R, E_{1} \cup E_{2}\right)$ gives the lower bound in Theorem 1 .

First, observe that $G$ has $z+z+(k-1)=n$ vertices and

$$
\begin{aligned}
\left|E_{1}\right|+\left|E_{2}\right| & =\frac{(n-k+1)(k+1)-4(1+2+\cdots+k / 2)}{2}+(n-k+1)(k-1) \\
& =(n-k+1)\left(\frac{3 k}{2}-\frac{1}{2}\right)-\frac{k(k+2)}{4}>\frac{3}{2}(k-1) n-2 k^{2}
\end{aligned}
$$

edges. It remains to show that $G$ contains no $k+1$ pairwise disjoint edges. Suppose that $D$ is a set of disjoint edges in $G$. If $\left|D \cap E_{1}\right| \leq 1$, then $|D| \leq|R|+1=k$. Otherwise, let the leftmost edge of $D \cap E_{1}, e_{1}$, intersect $r$ in a point $r_{s}$, and the rightmost one, $e_{2}$, in a point $r_{t}$. Since there are $s+k / 2$ vertices of $R$ to the left of $e_{1}, D$ contains at most $s+k / 2$ edges to the left of $e_{1}$. Similarly, $D$ contains at most $k / 2-t$ edges to the right of $e_{2}$. Since there are $t-s-2$ vertices of $P \cup Q$ between $e_{1}$ and $e_{2}, D$ contains at most $t-s-2$ edges between $e_{1}$ and $e_{2}$. Altogether, $D$ contains at most $(s+k / 2)+(k / 2-t)+(t-s-2)+2=k$ edges.

\section{The Case $k=3$}

\subsection{The Upper Bound}

We use the following result. 
Lemma 5 [GKK]. If a geometric graph $G$ of $n$ vertices does not contain four pairwise disjoint edges and there is a line which intersects every edge of $G$ and contains no vertex of $G$, then $G$ has at most $7 n$ edges.

Lemma 5 is not stated in [GKK] explicitly. However, its proof (relatively long caseanalysis) is readily contained in the proof of Theorem 2 in [GKK].

Let $G=(V, E)$ be a geometric graph without four pairwise disjoint edges. Denote the vertices by $v_{1}, \ldots, v_{n}$ from left to right, and assume that no pair of them lies on a vertical line. For any $1 \leq i<n$ let $\bar{G}_{i}$ be the subgraph of $G$ which contains only those edges $v_{\alpha} v_{\beta}$ of $G$ where $\alpha \leq i<\beta$.

It follows from Lemma 5 and the assumption that, for any $1 \leq i<n,\left|E\left(\bar{G}_{i}\right)\right| \leq 7 n$. For any $1 \leq i<n$, let $G_{i}^{-}$and $G_{i}^{+}$be the subgraph of $G$ induced by the vertices $v_{1}, \ldots, v_{i}$ and by $v_{i}, \ldots, v_{n}$, respectively. Let

$$
I=\max \left\{i \mid G_{i}^{-} \text {does not contain two disjoint edges }\right\} .
$$

Since $G_{I+1}^{-}$contains two disjoint edges, $G_{I+2}^{+}$does not. Suppose without loss of generality that $I<n / 2$. Then

$$
E(G)=E\left(G_{I}^{-}\right) \cup E\left(G_{I+2}^{+}\right) \cup E\left(\bar{G}_{I+1}\right) \cup\left\{v_{i} v_{I+1} \in E(G) \mid i \leq I\right\} .
$$

Therefore,

$$
|E(G)| \leq e_{1}(I)+e_{1}(n-I-1)+7 n+I<8.5 n .
$$

\subsection{The Lower Bound}

First, let $n$ be odd. Take the vertices of a regular $(n-2)$-gon, and join each of them with the furthermost four vertices. Add two vertices near the center of the $(n-2)$-gon, and join each of them by an edge to all the other vertices. The resulting geometric graph on $n$ vertices has $4(n-2) / 2+2(n-1)-1=4 n-7$ edges. It is easy to see that no four edges are pairwise disjoint, see Fig. 6.

For $n$ even, we take the above geometric graph on $n+1$ vertices, and remove a vertex and the six edges incident to it. The resulting graph on $n$ vertices has $4 n-9$ edges.

\section{Remarks}

By a little modification, it is possible to improve the upper bound of Theorem 1 slightly.

Theorem 6. For any $k \leq n / 2$,

$$
e_{k}(n) \leq \frac{4}{21} k^{3} n+O\left(k^{2} n\right) .
$$

Proof. (Sketch) We use the same partial orderings, $\prec_{1}, \prec_{2}, \prec_{3}, \prec_{4}$, as in the proof of Theorem 1 (see Fig. 1). For any edge $e$ of a geometric graph $G=(V, E)$, let 


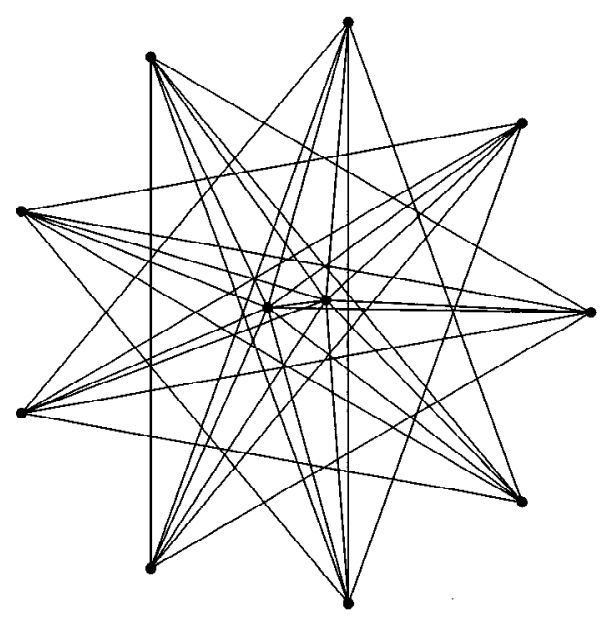

Fig. 6. A geometric graph with no four pairwise disjoint edges.

$\operatorname{Rank}_{3}^{+}(e)$ be the maximum number $a \geq 0$ such that there exist $e_{1}, e_{2}, \ldots, \prec e_{a} \in E$, $e \prec_{3} e_{1} \prec_{3} e_{2} \prec_{3} e_{3} \prec \cdots \prec_{3} e_{a}$, and let $\operatorname{Rank}_{4}^{-}(e)$ be the maximum number $b \geq 0$ such that there exist $e_{1}, e_{2}, \ldots, e_{b} \in E, e_{1} \prec_{4} e_{2} \prec_{4} e_{3} \prec \cdots \prec_{4} e_{b} \prec_{4} e$.

Lemma 7. Let $G=(V, E)$ be a geometric graph with $n$ vertices and with no $k+1$ pairwise disjoint edges. Suppose that there is a line which avoids all vertices and crosses all edges of $G$. Then

$$
|E| \leq \frac{1}{6} k^{3} n+O\left(k^{2} n\right) .
$$

Proof of Lemma 7. For any $0 \leq a, b \leq k-1$ let

$$
E_{a, b}=\left\{e \in E \mid \operatorname{Rank}_{3}^{+}(e)=a, \operatorname{Rank}_{4}^{-}(e)=b\right\} .
$$

Clearly, $E_{a, b}$ is an antichain with respect to $\prec_{3}$ and $\prec_{4}$. We can define $\overrightarrow{E_{a, b}}$ and its maximal zigzag paths just as in the proof of Theorem 1. It is easy to see that there are at most $n$ maximal zigzag paths and every edge of $\overrightarrow{E_{a, b}}$ is contained in at least one of them (in fact, in exactly one of them, but here we do not need that). Suppose that there is a zigzag path $\overrightarrow{e_{1}}, \overrightarrow{e_{2}}, \ldots, \overrightarrow{e_{x}}$ of $x=2(k-a-b)+1$ edges. Then $\overrightarrow{e_{1}}, \overrightarrow{e_{3}}, \overrightarrow{e_{5}}, \ldots, \overrightarrow{e_{x}}$ are $k-a-b+1$ pairwise disjoint edges. Moreover, we can add $a$ edges above $\overrightarrow{e_{1}}$ and $b$ edges below $\overrightarrow{e_{x}}$ to get $k+1$ pairwise disjoint edges, contradicting our assumption. (See Fig. 7.) Therefore, every maximal zigzag path of $\vec{E}_{a, b}$ has at most $2(k-a-b)$ edges, so

$$
\begin{gathered}
\left|E_{a, b}\right| \leq n(k-a-b), \\
|E|=\sum_{\substack{0 \leq a, b \\
a+b<k}}\left|E_{a, b}\right| \leq n \sum_{\substack{0 \leq a, b \\
a+b<k}}(k-a-b)=\left(\begin{array}{c}
k+2 \\
3
\end{array}\right) n .
\end{gathered}
$$




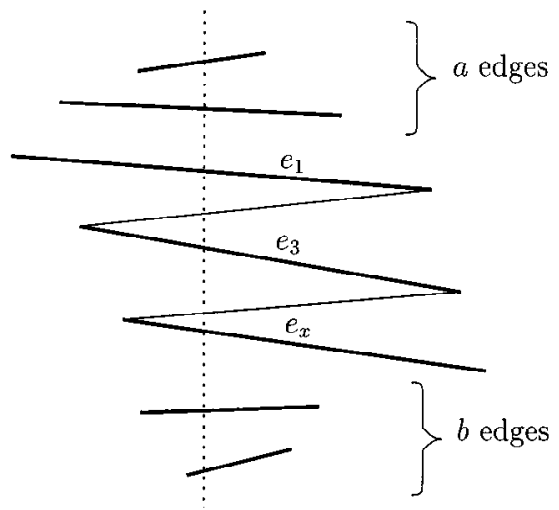

Fig. 7. $k+1$ disjoint edges.

Return to the proof of Theorem 6. It is not hard to see that there is a line $\ell$ which avoids all vertices of $G$ and on one side of $\ell$ there are $\lceil n / 2\rceil$ vertices and at most $\lceil k / 2\rceil$ pairwise disjoint edges, while on the other side of $\ell$ there are $\lfloor n / 2\rfloor$ vertices and at most $\lfloor k / 2\rfloor$ pairwise disjoint edges of $G$. We get the recursion

$$
e_{k}(n) \leq \frac{1}{6} k^{3} n+O\left(k^{2}\right) n+e_{\lceil k / 2\rceil}(\lceil n / 2\rceil)+e_{\lfloor k / 2\rfloor}(\lfloor n / 2\rfloor)
$$

and Theorem 6 follows.

If the number of edges in a geometric graph is at least $\Omega\left(n^{2}\right)$, then Theorem 1 guarantees $\Omega\left(n^{1 / 3}\right)$ pairwise disjoint edges. This is improved by the following result of Pach $[\mathrm{P}]$.

Theorem 8 [P]. For any $c>0$ there is a $c^{\prime}>0$ such that every geometric graph of $n$ vertices and at least $\mathrm{cn}^{2}$ edges has at least $c^{\prime} \sqrt{n}$ pairwise disjoint edges.

\section{References}

[AE] N. Alon and P. Erdős, Disjoint edges in geometric graphs, Discrete and Computational Geometry 4 (1989), 287-290.

[AH] S. Avital and H. Hanani, Graphs, Gilyonot Lematematika 3 (1966), 2-8 (in Hebrew).

[D] R. P. Dilworth, A decomposition theorem for partially ordered sets, Annals of Mathematics 51 (1950), 161-166.

[GKK] W. Goddard, M. Katchalski, and D. J. Kleitman, Forcing disjoint segments in the plane, European Journal of Combinatorics 17 (1996), 391-395.

[HP] H. Hopf and E. Pannwitz, Aufgabe No. 167, Jahresbericht der Deutschen Mathematiker-Vereinigung 43 (1934), 114.

[K] Y. Kupitz, Extremal Problems in Combinatorial Geometry, Aarhus University Lecture Notes Series, 53, Aarhus University, Denmark (1979).

[LMPT] D. Larman, J. Matoušek, J. Pach, and J. Törőcsik, A Ramsey-type result for planar convex sets, The Bulletin of the London Mathematical Society 26 (1994), 132-136. 
[MP] W. Moser and J. Pach, Recent developments in combinatorial geometry, in: New Trends in Discrete and Computational Geometry (J. Pach, ed.), Algorithms and Combinatorics, Vol.10, Springer-Verlag, Berlin, 1993, pp. 281-302.

[P] J. Pach, Personal Communication.

[PA] J. Pach and P. K. Agarwal, Combinatorial Geometry, Wiley, New York, 1995.

[PT] J. Pach and J. Törő́csik, Some geometric applications of Dilworth's theorem, Discrete and Computational Geometry 12 (1994), 1-7.

[T] G. Tóth, Note on geometric graphs (manuscript).

[V] P. Valtr, On geometric graphs with no $k$ pairwise parallel edges, Discrete and Computational Geometry 19 (1998), 461-469.

Received May 7, 1998, and in revised form March 24, 1999. 